\title{
Tasks for assessment of the episodic buffer: a systematic review
}

\author{
Alexandre de Pontes Nobre, Jaqueline de Carvalho Rodrigues, Juliana Burges Sbicigo, Luciane \\ da Rosa Piccolo, Maxciel Zortea, Sérgio Duarte Junior, and Jerusa Fumagalli de Salles
}

Universidade Federal do Rio Grande do Sul, Porto Alegre, RS, Brazil

\begin{abstract}
The aim of the present study was to review the tasks that have been used to assess the functioning of the episodic buffer in Baddeley's multicomponent model of working memory. A systematic review of studies published from January 2000 to February 2013 was conducted. The search term "episodic buffer" was used in the Web of Knowledge, PsycINFO, PubMed, Embase, and BVS-Psi databases. The selected articles consisted of empirical studies that used tasks to assess the episodic buffer. Theoretical and review papers and studies with animals were excluded. The final sample comprised 36 papers. The tasks were categorized as experimental tasks or standardized tests. The experimental tasks were grouped by modality (unimodal or crossmodal) and described according to four criteria: task to be performed, type of stimulus used, secondary task employed, if any, and retention interval. The standardized tests included classical measures of working memory. Some tasks were found not to meet experimental criteria that were needed to evaluate the episodic buffer. Moreover, some of the standardized tests did not provide theoretical arguments or empirical evidence that the episodic buffer is recruited to perform them. The results are discussed in the context of the multicomponent model of working memory. Keywords: working memory, episodic buffer, binding, experimental task, standardized test.
\end{abstract}

Received 30 October 2012; received in revised form 20 June 2013; accepted 27 June 2013. Available online 23 December 2013.

\section{Introduction}

Working memory (WM) is usually regarded as a framework for a series of interactive processes that comprise temporary storage and the manipulation of information (Baddeley, Allen, \& Hitch, 2011). In Baddeley and Hitch's (1974) original model, three components were proposed: a phonological loop, responsible for storing and maintaining phonological information; a visuospatial sketchpad, which stored and maintained visual and spatial information; and a central executive, which had a general storage capacity and was responsible for the manipulation of temporary information.

The storage capacity was subsequently removed from the central executive (Baddeley, \& Logie, 1999), which became a processing-only component. This led to some problems with the model, when it was observed that the short-term

Alexandre de Pontes Nobre, Jaqueline de Carvalho Rodrigues, Juliana Burges Sbicigo, Luciane da Rosa Piccolo, Maxciel Zortea, Sérgio Duarte Junior, and Jerusa Fumagalli de Salles, Universidade Federal do Rio Grande do Sul. Correspondence regarding this article should be directed to: Alexandre de Pontes Nobre, Universidade Federal do Rio Grande do Sul, Instituto de Psicologia, Rua Ramiro Barcelos, 2600, sala 114, Porto Alegre, RS, 90035-003, Brasil. E-mail: alpnobre@ gmail.com storage capacity of the phonological loop and visuospatial sketchpad (collectively known as "slave systems") was insufficient to explain human subjects' performance in a series of experimental tasks and complex cognitive activities (Baddeley, 2007, p. 141). Other problems were also raised, including the contribution of semantic factors to the recall of word sequences (Baddeley, 1966), the presence of substantial sentence span in some patients although their phonological short-term memory was impaired (Baddeley \& Wilson, 2002), the phenomenon of chunking (Baddeley, 2000; Baddeley, Hitch, \& Allen, 2009; Miller, 1956), and individual differences in the WM span of Daneman and Carpenter (1980).

To solve these issues, a fourth component was proposed: an episodic buffer (EB), conceived as a multidimensional storage system that integrates information from different sources, such as long-term memory (LTM), the slave systems, and perception. Access to the EB is achieved through consciousness. Therefore, the model provides a mechanism for multi-feature binding, which is regarded as a role of consciousness (Baddeley, 2000). In its latest version, the model contains connections between the EB, central executive, LTM, and both slave systems (Baddeley et al., 2011; Baddeley, 2012).

The introduction of this fourth component allowed WM to bind different information modalities. It also 
provided the system with a greater storage capacity, which might contribute to subjects' performance in verbal and visual tasks and tasks that demand the integration of multiple types of information (e.g. semantic, syntactic, and perceptual; Baddeley, 2007, pp. 148-151). As a component that allows syntactic and semantic information to interact with information held in short-term memory, the EB is also considered responsible for the phenomenon of chunking (Baddeley, 2007 , p. 150). A chunk integrates several items, such as words or numbers, into one representation, thus greatly expanding individuals' storage capacity. The EB is thought to be involved in memory for sentence and prose, which was a major focus of criticism of Baddeley and Hitch's original model (Repovs \& Baddeley, 2006).

At the time of its proposal (Baddeley, 2000), activity in the EB was considered to be subordinated to the central executive. This assertion was later modified, and two types of binding were proposed (c.f., Baddeley, 2007, p. 148). The first type was automatic binding, which should be independent of resources. The second type was active binding, which would rely on the central executive. This type of binding has been suggested to be involved in the integration of objects and crossmodal (e.g., visual/spatial and verbal) characteristics from the slave systems and LTM (Godoy \& Galera, 2011).

Since Baddeley postulated distinct types of binding in the EB (Allen, Baddeley, \& Hitch, 2006; Baddeley, 2007 , p. 171), a major goal of recent WM studies has been to determine which binding processes require executive resources. Most studies, however, have failed to find effects of attentionally demanding tasks on binding (Baddeley, 2012; Baddeley, Allen, \& Hitch, 2010). This led to a new revision of the model. The hypothesis in the reformulated WM model (Baddeley, 2012) is that the EB is an essentially passive storage component where bindings might be displayed and executive resources may carry out further manipulation. The binding of visuospatial or verbal information would occur outside WM or within the slave systems (Baddeley, 2012; Baddeley et al., 2010, 2011). In the case of crossmodal binding, however, evidence is less abundant. Some authors (Allen, Hitch, Mate, \& Baddeley, 2012; Elsley \& Parmentier, 2009; Godoy $\&$ Galera, 2011) have suggested that it depends on executive resources in a way that distinguishes it from the binding of information in single modalities.

Studies that aim at investigating the EB and its different types of binding have increased in number since its proposal. Nevertheless, despite the variety of tasks that are available to evaluate other components of $\mathrm{WM}$, the concept of the EB is still relatively new, and tasks that assess its function are still being developed with no comprehensive list of tasks being presently available. Therefore, it is important to systematize the tasks that have been used in studies that investigate this component and to determine how they assess its function. The aim of this study was to critically review the tasks that have been used to evaluate the function of the EB and how these tasks contribute to the investigation of the different types of binding proposed by Baddeley (2007, pp. 171-172). The theoretical background of the present systematic review was the latest version of the multicomponent model of WM (Baddeley, 2012).

\section{Methods}

A systematic review of papers published from January 2000 to February 2013 was conducted, for which the Web of Knowledge, PsycINFO, PubMed, Embase, and BVS-Psi databases were used. The search term "episodic buffer" was entered in the standard search field in all of the databases. Although this term is not yet available in the Medical Subject Headings or Thesaurus, it was considered the most adequate term for a comprehensive search that would achieve the aims of this review.

Papers were initially selected according to their abstracts to identify the studies that effectively met the aims. The following exclusion criteria were adopted: (i) theoretical and review papers, (ii) animal studies, and (iii) studies that did not explicitly aim at assessing the EB. The selection of papers and application of the exclusion criteria were performed by two independent judges. A third judge was consulted when discordances were observed. Once all of the studies that met the exclusion criteria were removed, the remaining papers were fully read to identify the tasks employed in the studies and the outcomes.

The tasks were separated into two categories: experimental tasks and tests. Experimental tasks were defined as those used in experimental studies that did not consist of or were part of any assessment instrument or battery. Tests were defined as tasks that consisted of or were part of any type of instruments with established psychometric properties, such as standardization or normative studies (Strauss, Sherman, \& Spreen, 2006).

Experimental tasks were organized in terms of the modality of information used: unimodal tasks (further subdivided into visual/spatial and verbal tasks) and crossmodal tasks. The reason for this differentiation lay in the different areas of research that investigate binding processes in each specific WM component (e.g., Allen et al., 2006; Baddeley et al., 2009) and across components (e.g., Elsley \& Pamentier, 2009). Tasks in each category were analyzed according to the following experimental characteristics: task type, stimulus material, secondary task employed, and retention interval (RI). Standardized tests are presented as a separate topic in the Results section below. The same structure was adopted in the Discussion section, where a critical analysis of the tasks is presented, emphasizing the types of binding evaluated by each task.

\section{Results}

Thirty-six empirical studies were selected that explicitly aimed at using specific tasks and tests to assess EB function in human subjects. The study selection flowchart is presented in Figure 1. 


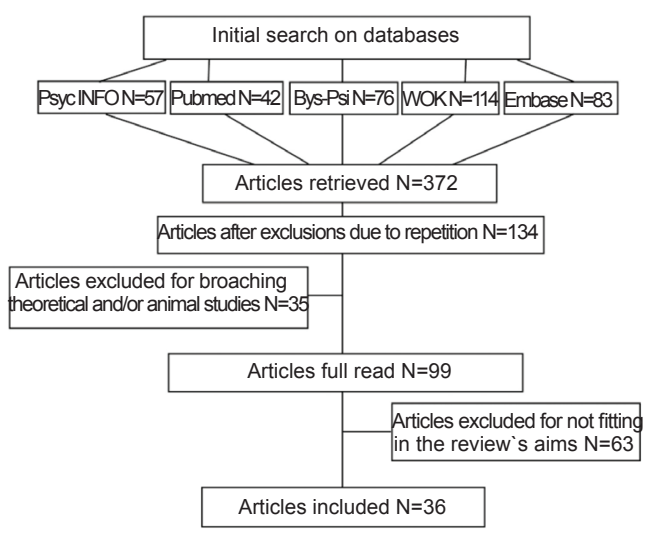

Figure 1. Studies selection process flowchart

\section{Experimental tasks}

Table 1 presents unimodal experimental tasks according to the modality of information used (i.e., verbal and visual/spatial). Task type, stimulus material, secondary task employed, and RI are indicated for each experiment.

\section{Unimodal tasks}

Visual/spatial tasks. In a total of seven papers analyzed, visuospatial binding processes were evaluated through recognition, recall, and spatial visual search tasks. The stimuli used in these tasks comprised geometric shapes, colors, and drawings of real objects.

Recognition tasks varied as a function of stimulus type and exposure duration during the study, delays between the study and test phases, and the number of response choices (yes/no or more options). Karlsen, Allen, Baddeley, and Hitch (2010) presented participants with a single-probe recognition task, in which each trial consisted of the presentation of a stimulus (e.g., shape and color, which varied from 250 to $3,000 \mathrm{~ms}$ ) for study, a delay (often $900 \mathrm{~ms}$ ), and a yes/no recognition phase.

In the study by Allen et al. (2006; experiments 1 and 5), the exposure time of the stimuli during the study phase was kept at $250 \mathrm{~ms}$. Parra, Sala, Logie, \& Abrahams (2009; experiment 1) used a similar yes/no recognition paradigm, with participants requested to answer whether the test probe was the same or different than the study stimuli. A $900 \mathrm{~ms}$ delay between the study and test phases was used, with an exposure duration of 2,000 ms per study item. In experiments 3 and 4 in the study by Parra et al. (2009), nameable objects were used instead of abstract figures. The stimulus duration was reduced to $1,500 \mathrm{~ms}$ per item (the number of items varied between two and six), and no RI was employed. Additionally, in each trial in experiment 3 , the participants had to mark on a multiple-choice recognition list the items that had been presented in the study phase.

Mate, Allen and Baqués (2012) also used a multiplechoice test with an exposure duration of $4,000 \mathrm{~ms}$ in the study phase. Most of these studies used conditions that included shape only, color only, either shape or color, and combinations of shape and color. Karlsen et al. (2010) also spatially or temporally separated these characteristics. Ally, Simons, McKeever, Peers and Budson (2008) used a different recognition memory paradigm, in which all of the stimuli (colored drawn objects) were studied for 2,000 ms each, followed by a $10 \mathrm{~min}(600,000 \mathrm{~ms}) \mathrm{RI}$ and a test phase in which the stimuli were presented from the same or a different perspective as the studied object. The participants responded whether the stimuli had been presented in the study phase.

The free recall task in Parra et al. (2009; experiment 6) consisted of the presentation of drawings of geometrical objects in the test phase, followed by the same conditions used in other studies (i.e., color only, shape only, and color-shape binding). The spatial visual search task (Kessels, Meulenbroek, Fernández, \& Olde Rikkert, 2010) consisted of opening virtual boxes on a screen to find where the probe object was. Errors in the search in the subsequent trials (i.e., choosing a box that had already been opened and did not contain the target object) were scored.

Some papers reported the use of secondary tasks to examine the role of attentional resources in binding processes. Backward counting consisted of presenting a random number to the participant, who then was asked to count aloud backward (either in ones or threes) from that number. Articulatory suppression consisted of repeating aloud a given number or pairs of words (Mate et al., 2012, used pairs with different semantic aspects). Both types of secondary tasks were performed at different moments of the task (e.g., during encoding and retrieval, during retention only, or during encoding and retention).

Allen et al. (2006) used digit span recall as a concurrent task during encoding and retention. This task consists of recalling an array of six digits in the order they were presented. Importantly, two studies did not explicitly instruct the participants in the study phase to hold both visuospatial code features online during the task, although in Kessels et al. (2010), the search task automatically implied that the subject needed to know what (figure) and where (location) the object was to answer correctly.

Verbal tasks. Ten studies were analyzed that used verbal tasks to evaluate the EB. In contrast to the visual modality, the experimental tasks that employed verbal material consisted mainly of recall tasks, sentence judgment, and 2-back tasks. None of the studies employed recognition tasks.

Verbal tasks exhibited little variation with regard to the time of exposure to the stimuli in the study phase and RI. Most of the tasks did not include a RI. Exceptions included the sentence judgment task by Newman, Malaia, Seo and Cheng (2012), which employed a RI of $6,000 \mathrm{~ms}$; the immediate free recall task in the study by Parra et al. (2009), in which an interval of 1,000 ms was used; and the 2-back task in the study by Rudner, Fransson, Ingvar, Nyberg and Rönnberg (2007), who used intervals that varied between 3,000 and 28,000 ms. 
Table 1. Unimodal experimental tasks organized by task type, stimulus type, use of secondary tasks, and retention intervals.

\begin{tabular}{|c|c|c|c|c|}
\hline Paper & Task & $\begin{array}{l}\text { Stimulus types } \\
\text { to bind }\end{array}$ & Secondary Task & Retention interval \\
\hline \multicolumn{5}{|c|}{ Visual/spatial tasks } \\
\hline Ally et al. $(2008)^{a}$ & Delayed yes/no recognition & Colored pictures of objects & - & $600,000 \mathrm{~ms}$ \\
\hline Allen et al. (2006) & Immediate yes/no recognition & Shapes and colors & $\begin{array}{l}\text { AC (numbers; Exp. } 1 \text { and } \\
\text { 5), BC (Exp. 2), digit span } \\
\text { recall (Exp. 3), and BC } \\
\text { (Exp. 4) }\end{array}$ & $900 \mathrm{~ms}$ \\
\hline Allen et al. (2012) & Immediate yes/no recognition & Shapes and colors & AS (numbers) and BC & $1,000 \mathrm{~ms}$ \\
\hline Karlsen et al. (2010) & Immediate yes/no recognition & Shapes and colors & AS (numbers) and BC & $\begin{array}{l}900 \mathrm{~ms} \text { (exp. 1-6) } \\
\text { and } 500 \mathrm{~ms} \text { (exp. 7) }\end{array}$ \\
\hline Kessels et al. (2010) & Spatial visual search & $\begin{array}{l}\text { Spatial locations, objects, } \\
\text { and colors }\end{array}$ & - & Does not apply \\
\hline Mate et al. (2012) & $\begin{array}{l}\text { Immediate multiple-choice } \\
\text { recognition }\end{array}$ & Shapes and colors & AC (word pairs) & $1,000 \mathrm{~ms}$ \\
\hline Parra et al. (2009) Exp. 1 & Immediate yes/no recognition & Shapes and colors (Exp. 1) & - & $900 \mathrm{~ms}$ \\
\hline $\begin{array}{l}\text { Parra et al. (2009) Exp. } \\
3 \text { and } 4\end{array}$ & $\begin{array}{l}\text { Immediate multiple-choice } \\
\text { recognition (Exp. 3), immediate } \\
\text { yes/no recognition (Exp. } 4 \text { ) }\end{array}$ & $\begin{array}{l}\text { Nameable objects and } \\
\text { colors }\end{array}$ & - & None \\
\hline Parra et al. (2009) Exp. 6 & Immediate free recall & Shapes and colors & 一 & None \\
\hline \multicolumn{5}{|c|}{ Verbal tasks } \\
\hline Alloway et al. (2004) & Immediate recall & Sentences & - & None \\
\hline $\begin{array}{l}\text { Baddeley et al. (2009) } \\
\text { Exp. 1, 2, and } 3\end{array}$ & Immediate serial recall & $\begin{array}{l}\text { Constrained sentences and } \\
\text { word lists }\end{array}$ & $\begin{array}{l}\text { AS and visuospatial CRT } \\
\text { (Exp. 2), verbal and visu- } \\
\text { ospatial n-back (Exp. 3) }\end{array}$ & None \\
\hline $\begin{array}{l}\text { Baddeley et al. (2009) } \\
\text { Exp. } 4\end{array}$ & Delayed serial recall & $\begin{array}{l}\text { Constrained sentences and } \\
\text { word lists }\end{array}$ & $\mathrm{AS}$ and $\mathrm{BCT}$ & $2,000 \mathrm{~ms}$ \\
\hline Belacchi et al. (2011) & Sentence judgment & Sentences & - & None \\
\hline Germano et al. (2008) & $\begin{array}{l}\text { Immediate and delayed free } \\
\text { recall }\end{array}$ & Word lists & Sequential crossing of boxes & None \\
\hline $\begin{array}{l}\text { Hoffman, Jefferies, } \\
\text { Ehsan, Jones, \& Lambon } \\
\text { Ralph (2012) }\end{array}$ & Immediate serial recall & Sentences and word lists & - & None \\
\hline $\begin{array}{l}\text { Jefferies et al. (2004) } \\
\text { Exp. } 1\end{array}$ & Delayed serial recall & $\begin{array}{l}\text { Word lists, unrelated } \\
\text { sentences }\end{array}$ & Visuospatial CRT & $20,000 \mathrm{~ms}$ \\
\hline $\begin{array}{l}\text { Jefferies et al. (2004) } \\
\text { Exp. } 2 \text { and } 3\end{array}$ & $\begin{array}{l}\text { Immediate serial recall (Exp. } 2 \\
\text { and 3) }\end{array}$ & $\begin{array}{l}\text { Word lists, unrelated sen- } \\
\text { tences, and stories (Exp. 3) }\end{array}$ & Visuospatial CRT & None \\
\hline $\begin{array}{l}\text { Kapikian, \& Briscoe } \\
(2012)\end{array}$ & Immediate serial recall & Stories and sentence lists & Visuospatial CRT & None \\
\hline Newman et al. (2012b) & Sentence judgment & Sentences & - & $6,000 \mathrm{~ms}$ \\
\hline $\begin{array}{l}\text { Parra et al. (2009) } \\
\text { Exp. } 5\end{array}$ & Immediate free recall & Nouns and adjectives & - & $1,000 \mathrm{~ms}$ \\
\hline Rudner et al. (2007) & 2-back task & $\begin{array}{l}\text { Sign language and speech } \\
\text { lexical items }\end{array}$ & - & $3,000-28,000 \mathrm{~ms}$ \\
\hline
\end{tabular}

BC, backward counting; CRT, continuous reaction time task; AS, articulatory suppression; ${ }^{\text {a }}$ event-related potentials experiment; ${ }^{\mathrm{f}}$ fMRI experiment

Exposure times to the stimuli of 500-2,000 ms per word were adopted. Rudner et al. (2007) did not report the exposure duration in their study.

The material used in these experiments comprised words, sentences, and stories. The type of information (syntactic and semantic) that could be used to process the stimuli was manipulated. Some studies used sentences as stimuli, which varied in the degree to which they demanded syntactic or semantic contributions from LTM (Belacchi, Benelli, \& Pantaleone, 2011; Newman et al., 2012). Other studies compared word lists that were either semantically clustered lists (i.e., composed of organized clusters of four words from each of three semantic categories that were presented consecutively) or unclustered lists (i.e., composed of unrelated words; Germano, Kinsella, Storey, Ong, \& Ames, 2008). In these tasks, sentences or word lists that could benefit from semantic or syntactic contributions from LTM should exhibit a processing advantage in the form of chunking. Because the EB in its original proposal (Baddeley, 2000; Baddeley, 2007, pp. 149-150) was considered responsible for these types of bindings, this processing advantage was assumed to reflect EB activity. Therefore, investigating variables that affect the functioning of the EB would be possible by examining which variables impact verbal binding in those tasks.

Other tasks used different types of stimuli to compare distinct types of chunking. Jefferies, Lambon Ralph and Baddeley (2004) compared the recall of unrelated words, words in sentences, unrelated sentences, and stories. The rationale behind these tasks was that material that allowed subjects to use more 
syntactic and semantic information to be recalled or judged would rely more on the EB, similar to tasks that use only sentences or word lists. In the case of the task used by Jefferies et al. (2004), unrelated sentences could benefit from contributions from LTM to a lesser degree than thematically related sentences (as in the case of stories) or word lists.

Kapikian and Briscoe (2012) used a similar task but attempted to control the variables involved in within-sentence processing by comparing the same set of sentences under two conditions: coherent and incoherent. In the coherent condition, sentences were ordered to constitute a story. In the incoherent condition, they were reordered such that the global coherence of the story was removed, effectively turning the set into a series of unrelated sentences. According to the authors, stories would benefit more from contributions from LTM, in a similar way as the task used by Jefferies et al. (2004).

Baddeley et al. (2009) compared the recall of words and sentences. However, because memory of sentences is under the influence of a series of variables, such as semantic memory and sentence length, the authors developed a new task to control these differences. The main variable that differentiated the sentences and word lists was sequential redundancy. For this task, referred to as constrained sentence span, sentences were created from the same words used in the word lists. This led to a repeated presentation of words across trials, leading to proactive interference, which should minimize the contribution of long-term episodic and semantic memory. Additionally, in experiment 1, because the sentences did not contain function words, they were not strictly grammatical. Function words were subsequently added in experiment 2. Thus, the sentences would contain the same number of words as the word lists, differing only in the sequential redundancy effect. Coupled with proactive interference, this would control for the effects of semantic and episodic memory and sentence length, forcing the participants to rely on binding in WM for recall.

Rudner et al. (2007) used a different paradigm in which the binding of distinct types of linguistic modalities, namely sign language and speech lexical items, was explored. They compared performance in sign-speech bilingual participants in a 2-back task, in which stimuli were presented in one of two modalities: speech or sign language. In two conditions, speech only and sign language only, the participants were instructed to respond in the same modality in which the stimuli were presented. In a third binding condition, the stimuli were presented in one modality (e.g., speech), and the participant responded in the other modality (e.g., sign language).

Studies that used dual-task paradigms employed articulatory suppression (AS), continuous reaction time (CRT), and n-back tasks to evaluate the role of executive processes and slave systems (phonological loop and visuospatial sketchpad) in binding. Jefferies et al. (2004) investigated the effect of attention on the binding of words, sentences, and stories using CRT concomitantly with immediate and delayed serial recall of the study material. Baddeley et al. (2009; experiment 2) also used a CRT task as a secondary task but considered that it disrupted the functioning of both the visuospatial sketchpad and central executive; therefore, they combined it with AS to prevent the use of phonological aids by the participants. In the third experiment, visual and verbal versions of the n-back task, with two lags (0-back and 2-back), allowed for more systematic comparisons of WM components, with the 0-back lags essentially taxing the phonological loop and visuospatial sketchpad, whereas the 2-back lags also consumed executive resources.

Germano et al. (2008) manipulated a series of word lists to explore the impact of conditions of full and divided attention on the strategic chunking or clustering of information, which is supposed to be associated with the EB. In the divided attention condition, the participants performed a task in which they had to mark a cross inside boxes that were linked to form an irregular path laid out on a sheet of paper (cf., Baddeley, 1996). The authors did not consider the possible disruption of the visuospatial sketchpad in this task, instead assuming that it principally taxed the central executive.

\section{Crossmodal tasks}

A total of 10 studies were found that used tasks that aimed at investigating binding across modalities (i.e., the binding of verbal and visuospatial material). Table 2 lists the tasks and their characteristics.

Crossmodal tasks consisted of immediate yes/ no recognition and immediate recall tasks. The experimental procedures varied in different ways across studies. Stimulus exposure times ranged from 1,000 to $5,000 \mathrm{~ms}$, and RIs varied between 950 and 20,000 ms in immediate yes/no recognition tasks (Table 2). Studies that employed recall tasks did not include RIs (Piolino et al., 2010; Quinette et al., 2006; Zhang et al., 2004).

Immediate recognition tasks involved binding between letters and spatial locations (Elsley \& Parmentier, 2009; Harkin \& Kessler, 2009; Luck, Danion, Marrer, Pham, Gounot, \& Foucher, 2010a, b; Piolino et al., 2010; Poch et al., 2010; Quinette et al., 2006), words and spatial locations (Campo et al., 2008), and faces and names (Godoy \& Galera, 2011). Recall tasks also involved binding between letters and spatial locations (Piolino et al., 2010; Quinette et al., 2006) and between auditory digits and spatial locations (Zhang et al., 2004).

In five of the recognition paradigms (Elsley \& Parmentier, 2009; Harkin \& Kessler, 2009; Luck et al., 2010a, b; Poch et al., 2010), the binding condition consisted of the presentation of letters at spatial locations during the study phase. In the test phase, the subjects had to recognize whether the single letter was presented in the same position or in a different position from the one during the study phase. Quinette et al. (2006) 
Table 2. Crossmodal experimental tasks organized by task type, stimulus type, use of secondary tasks, and retention intervals.

\begin{tabular}{|c|c|c|c|c|}
\hline Paper & Task & Stimulus types to bind & Secondary task & Retention interval \\
\hline Campo et al. (2008) & $\begin{array}{l}\text { Immediate yes/no } \\
\text { recognition }\end{array}$ & Words and spatial locations & - & $2,500 \mathrm{~ms}$ \\
\hline Elsley, \& Parmentier (2009) & $\begin{array}{l}\text { Immediate yes/no } \\
\text { recognition }\end{array}$ & Letters and spatial locations & Three pure tones & $950 \mathrm{~ms}$ \\
\hline Godoy, \& Galera (2011) & Delayed recognition & Names and pictures of human faces & BCT & $6,000 \mathrm{~ms}$ \\
\hline Harkin, \& Kessler (2009) & $\begin{array}{l}\text { Immediate yes/no } \\
\text { recognition }\end{array}$ & Letters and spatial locations & - & $\begin{array}{l}500 \mathrm{~ms} \text { (probe 1) } \\
1,000 \mathrm{~ms} \text { (probe 2) }\end{array}$ \\
\hline Luck et al. (2010a) & Delayed recognition & Letters and spatial locations & - & $9,500 \mathrm{~ms}$ \\
\hline Luck et al. (2010b) & Delayed recognition & Letters and spatial locations & - & $9,500 \mathrm{~ms}$ \\
\hline Piolino et al. (2010) & $\begin{array}{l}\text { Immediate or delayed } \\
\text { recognition } \\
\text { Immediate recall }\end{array}$ & $\begin{array}{l}\text { Letters, colors, and } \\
\text { spatial locations } \\
\text { Letters and spatial locations }\end{array}$ & - & $\begin{array}{l}1,000,8,000, \text { or } \\
20,000 \mathrm{~ms} \\
\text { Does not apply }\end{array}$ \\
\hline Poch et al. $(2010)^{\mathrm{a}}$ & $\begin{array}{l}\text { Immediate yes/no } \\
\text { recognition }\end{array}$ & Letters and spatial locations & - & $1,200 \mathrm{~ms}$ \\
\hline \multirow[t]{2}{*}{ Quinette et al. (2006) } & $\begin{array}{l}\text { Immediate or delayed } \\
\text { recognition }\end{array}$ & $\begin{array}{l}\text { Letters, colors, and } \\
\text { spatial positions }\end{array}$ & - & 1,000 or $8,000 \mathrm{~ms}$ \\
\hline & Immediate recall & Letters and spatial locations & & Does not apply \\
\hline Zhang et al. (2004) ${ }^{\mathrm{b}}$ & Immediate serial recall & $\begin{array}{l}\text { Auditory digits and } \\
\text { visual locations }\end{array}$ & - & None \\
\hline
\end{tabular}

and subsequently Piolino et al. (2010) used a binding condition in which colored letters were presented in the center of a grid along with randomly placed crosses that matched the letters in color. In the test phase, the subjects were presented with one of the previous letters in black, which did or did not match the position of the crosses. The subjects were asked to answer whether the matching was correct. In the binding condition used by Campo et al. (2008), ellipses were presented at random locations on a screen in the study phase. Each ellipse contained a word. In the test phase, the subjects were presented with the name of a semantic category within an ellipse and had to decide whether one of the words studied belonged to that category and whether the location of the ellipse was the same as in the study phase. In the study by Godoy and Galera (2011), the binding of visual and verbal information was assessed in a condition in which the subjects learned names and human faces in combination. The subjects then had to recognize whether the combination was the same as the one in the study phase.

The immediate recall task used by Piolino et al. (2010) and Quinette et al. (2006) consisted of a series of combined verbal/spatial spans of increasing lengths, ranging from three to 11 items. The items were composed of consonants in distinct spatial locations, and the subjects were required to memorize the order. Immediately after each presentation, they had to recall each consonant orally while tapping the correct location on a blank grid. In the recall task employed by Zhang et al. (2004), the participants were presented sequences of three or five digits through earphones and three or five locations. Afterward, they had to remember the items with finger gestures and their spatial location by pointing their index finger at the corresponding positions on the panel.

Some of the aforementioned studies (Elsley \& Parmentier, 2009; Godoy \& Galera, 2011) used secondary tasks to investigate whether crossmodal binding demanded executive resources. In the study by Elsley and Parmentier (2009), binding between letters and spatial locations was investigated in a condition in which a concurrent three-pure-tone task was presented via headphones after each trial. The participants were not instructed to memorize the connection between the two features. Godoy and Galera (2011) requested that the participants perform a Backward Counting by Threes (BCT) task during the RI between the study and test phases of the primary recognition task. Both studies used secondary tasks that employed material with different codes (i.e., auditory and numeric) from the codes used in the primary tasks to control for the effects of interference in the same components of WM recruited during the primary tasks (Elsley \& Parmentier, 2009).

\section{Standardized tests}

Eleven studies were found which used standardized tests to assess the EB. The following tests were employed to evaluate the functioning of the EB by using words as material: Test of Memory and Learning-Paired recall (TOMAL), Recalling Sentences task from the Clinical Evaluation of Language Fundamentals, 4th edition (CELF-4), California Verbal Learning Test (CVLT), 
and Rey Auditory Verbal Learning Test (RAVLT) immediate and delayed recall (Allen et al., 2010; Henry, 2010; Martins, \& Ortiz, 2009).

In the Paired Recall task from the TOMAL, subjects are asked to learn pairs of words, half of which are semantically associated. The Recalling Sentences task from the CELF-4 (Petruccelli, Bavin, \& Bretherton, 2012) is a sentence repetition task in which words are presented orally to the participant, who is instructed to repeat them. The CVLT comprises the presentation of two word lists. List A is read aloud five times to the participant, who proceeds to recall as many words as possible, in any order, after each presentation. List B, an interference list, is then presented orally, followed by its free recall. The participant is then prompted to recall the words from List A without hearing it again, under two conditions: immediate free recall and cued recall (using categories as cues). A delay of approximately 20 to $30 \mathrm{~min}$ follows, after which the participants have to retrieve the words from List A in three tasks: free recall, cued recall, and recognition. Finally, following a new delay of $15 \mathrm{~min}$, the subject performs forced-choice recognition of the words. The RAVLT is similar to the CVLT but does not include the cued recall tasks nor the last recognition task.

The immediate and delayed prose recall task from the Wechsler Memory Scale (WMS) and the Test of Memory and Learning-Memory for Stories were used to assess the EB at the sentence level (Altgassen, Phillips, Kopp, \& Kliegel, 2007; Baddeley \& Wilson, 2002; Berlingeri et al., 2008; Gooding, Isaac, \& Mayes, 2005; Henry, 2010; Vaz, Pradella-Hallinan, Bueno, \& Pompéia, 2011). Both consist of reading a story, followed by the assessment of the memory of that story.

Category Fluency (semantic and phonemic) evaluates executive skills (Henry, 2010; Price et al., 2010). The Biber Figure Learning Test-Expanded (BFLT-E) employs visual stimuli (Allen et al., 2010). The Operation-word Span Test and Visuospatial Maintenance Task (Tudesco et al., 2010) are used as measures of EB function. In the verbal fluency task, subjects are asked to retrieve as many words as possible according to a specific criterion (e.g., words beginning with a certain letter for phonemic fluency or belonging to a category for semantic fluency). This test was used as a measure of WM capacity.

The BFLT-E is designed to be a visual analog of the CVLT (Glosser, Cole, Khatri, DellaPietra, \& Kaplan, 2002), using geometric figures instead of words. The test comprises a series of five learning trials, an interference trial, an immediate recall condition, a delayed recall condition, and a cued recall condition. In the Operationword Span Task, sets of arithmetical operations and word strings are presented, followed by a question mark that indicates the moment at which the participant must classify the statement as right or wrong and then read the subsequent word aloud. After several strings, subjects are asked to recall all of the words presented in any order.
The Visuospatial Maintenance task consists of the presentation of a matrix on a computer screen where random sequences of digits appear one at a time in random locations. Following the presentation of sequences, question marks are shown, one by one, in the cells in which the digits were previously presented. The participants are required to recall the number that appeared in the location of the question mark.

\section{Discussion}

The aim of the present study was to review the tests and experimental tasks used to evaluate the functioning of the EB with regard to its role in the binding of information from a number of different sources, both within and across modalities. In its original proposal (Baddeley, 2000), the EB was itself responsible for binding the information. In more recent papers (e.g., Baddeley, 2012), the EB is regarded as a passive storage component where bindings performed elsewhere are maintained for further manipulation. Additionally, the present review investigated how these tasks contribute to the investigation of the different types of bindings that might be stored in the EB. According to Henry (2010), no clear methodological agreement exists for assessing the EB independently. Baddeley (2012) indicated that measurement of the EB is an unresolved problem. The tasks examined in this review were selected on the basis of theoretical explanations provided by the authors and are discussed below.

\section{Unimodal tasks}

Visual/spatial tasks. Six of the seven studies that investigated the EB using visuospatial tasks employed recognition tasks (Allen et al., 2006, 2012; Ally et al., 2008; Karlsen et al., 2010; Mate et al., 2012; Parra et al., 2009). Although in all of them the participants were evaluated based on their ability to recognize whether the probe stimulus was present in the study phase, they used distinct types and numbers of visual stimuli (e.g., geometric shapes and drawings of real objects) and therefore distinct levels of stimulus complexity. This could have led to the evaluation of slightly different characteristics of the EB. Stimuli such as those used by Kessels et al. (2010; i.e., colored real figures) might be processed differently from those used by Parra et al. (2009; i.e., geometrical shapes). The former stimuli could recruit verbal or LTM information, whereas the latter stimuli appear to be more visually dependent. The number of choices available in the test phase might also place different demands on the response, and this could restrict comparisons of the results.

Allen et al. (2006) and Karlsen et al. (2010) used the same type of recognition task (i.e., single recognition probe). Based on this design, the authors conducted a series of experiments, most of them imposing binding processes of color-shape, temporal, and spatial characteristics, and attentionally demanding aspects. Parra et al. (2009) also used this type of recognition task and were able to investigate the contributions of 
verbal information (i.e., nameable objects) to binding processes through experimental manipulations.

The task used by Ally et al. (2008) was a novel recognition paradigm. This task seems suitable for the investigation of how attentional processes are involved in the retrieval of information stored in WM because the probe stimulus changes according to the position of the original (study) item. However, Ally et al. (2008) used a 10 min delay between the study and test phases, which raises the question of whether such a long RI does not lead to an evaluation of LTM instead of WM and shortterm storage components.

Baddeley (2012) presented studies of visual binding, and most of them employed very short delays in the study and test phases (e.g., 300-9,000 ms according to Phillips \& Baddeley, 1971). The visuospatial search task utilized by Kessels et al. (2010) appears to be a more ecologically valid task (visual search for objects in the visuospatial field), but other variables, such as attentional demands or binding processes, were not strictly manipulated.

Future studies could focus on tasks that more closely resemble daily activities, the manipulation of binding processes between more similar stimuli (e.g., visual and spatial information), and the relationship between these processes and executive resources. So far, the experiments that assessed the binding of visuospatial information led Baddeley (Allen et al., 2012; Baddeley, 2012) to assume that the binding of visuospatial information is automatic, thus not relying on the central executive. However, as Baddeley et al. (2011) pointed out, most of those experiments investigated the binding and maintenance of features in the presence of strong perceptual support, as in the case of the binding of shape and color, which could be achieved by perceptual processes. Binding in the absence of strong perceptual support, in contrast, might demand more executive resources than the binding of simple features described in the studies in the present review. Findings in this field could contribute to the understanding of the role of the EB in clinical conditions.

The use of visual stimuli in short-term memory tasks and standardized tests has been criticized because the participants are able to make use of phonological coding to enhance their performance (Brandimonte, Hitch, \& Bishop, 1992). Some of the studies in the present review devised strategies in which the stimuli were processed mainly through the visuospatial sketchpad (otherwise, it could result in cross-modal binding processes). This could be achieved, for example, by instructing the participants to perform an articulatory suppression task to avoid the use of verbal coding (e.g., Karlsen et al., 2010). Parra et al. (2009) employed a change detection paradigm that is supposed to decrease the influence of verbal processing. Nevertheless, visual items used by Ally et al. (2008; i.e., drawn real figures) may have a limitation in relation to this issue.

Verbal tasks. One of the main problems with the previous WM model was the larger capacity for prose and sentence recall compared with the recall of digits, letters, or single words. According to Baddeley and Wilson (2002), at the core of the problem was the relationship between short-term memory storage components (i.e., the phonological loop and visuospatial sketchpad) and LTM, because comprehension involved combining representations in LTM. Baddeley and Wilson (2002) examined prose recall in amnesic patients and concluded that performance in this task was an index of EB function. Accordingly, experimental tasks developed since Baddeley and Wilson's study manipulated the need for semantic or syntactic information in the processing of linguistic material.

In all of these tasks, performance was compared across conditions in which information in LTM differentially contributed to the task. As the EB is deemed to be responsible for the binding of verbal material and information from LTM, these studies assumed that those conditions demanded more or less involvement from the EB. However, a large number of variables may be important for comprehension, which led Baddeley et al. (2009) to suggest the need to better control for differences between different types of stimuli in verbal tasks.

An important distinction concerns the type of task to be used. Recognition and recall of verbal stimuli tend to vary in the degree to which they rely on integration with LTM, depending on the kind of material that is employed. Performance on sentence judgment tasks, in contrast, is thought to draw heavily on binding with information in LTM (Belacchi et al., 2011). This also appears to be the case with the 2-back task used by Rudner et al. (2007). These differences should be considered when devising experimental tasks to evaluate the EB.

Notably, verbal binding is theoretically considerably less specified than visual binding, even though more studies were found that explored verbal binding (10) than visual binding (seven). One reason for this might be the fact that Baddeley himself has focused on visual binding (Baddeley et al., 2011). Consequently, the role of the phonological loop in verbal binding is less clear than what is known about the relationship between the visuospatial sketchpad and visual binding. In the study by Baddeley et al. (2009), performing articulatory suppression or a verbal 2-back task (which places demands on the phonological loop) did not differentially impair the recall of sentences and word lists, which led the authors to conclude that the phonological loop does not contribute to binding. Baddeley et al. (2012) stated that verbal binding occurs automatically in LTM, but this does not include the phonological loop in the explanation. However, some studies (e.g., Newman et al., 2012) posited a temporal role for the phonological loop in verbal binding.

Rudner et al. (2007) investigated the binding of two verbal modalities: sign language and speech. Based on previous research that showed that both modalities have at least partially distinct representations, the authors hypothesized that the 
binding condition should require the formation of unitary multidimensional representations of different types of verbal information. This type of binding differs from the one that is assessed in crossmodal tasks because the binding between sign language and speech, according to the authors, occurs between information in the phonological loop, LTM, and a "sign loop," which performs functions that are analogous to the phonological loop for sign language.

Based on existing studies, a framework of primary binding tasks and concurrent (secondary) tasks may be formulated that allows the investigation of the relationship between verbal binding and attentional demands on the central executive. In most cases, little evidence supports the involvement of the central executive in verbal binding. Jefferies et al. (2004) used a concomitant CRT task to compare the effects of attentional load on the recall of words in lists, words in sentences, unrelated sentences, and stories. The recall of words in sentences and stories was not impaired to a greater extent than the binding of words in lists, which suggested that verbal binding does not rely on the central executive and is performed automatically. Baddeley et al. (2009) found no differential impairment in the recall advantage for constrained sentences than for word lists by taxing the central executive, suggesting that the binding of words in sentences is automatic, thus corroborating the results of Jefferies et al. (2004). Kapikian and Briscoe (2012) compared chunking through the recall of coherent (binding) and incoherent conditions in subjects who performed a concomitant CRT task and under baseline conditions. Consistent with the results of Jefferies et al. (2004) and Baddeley et al. (2009), the binding condition did not suffer more from the division of attention caused by performing the CRT task compared with the incoherent condition, suggesting that the integration of sentences in stories is relatively automatic.

Jefferies et al. (2004), however, found a differential impact of a secondary attentionally demanding task on the recall of unrelated sentences compared with word lists and stories, presumably because unrelated sentences benefit less from the contribution of LTM and thus demand more from the central executive. Germano et al. (2008) compared the learning of semantically clustered and unclustered word lists under conditions of full and divided attention to investigate whether the chunking of words is impaired when attention is divided. They found that chunking was indeed compromised by the secondary task, suggesting that it relies at least partially on central executive resources.

Thus, prose recall and the chunking of words in sentences appear to rely on LTM and be automatic. On the other hand, the binding of unrelated sentences and chunking of words in word lists appear to demand considerably more executive resources, the type of binding that Baddeley $(2007$, p. 172) referred to as active binding. This distinction highlights the importance of considering the type of material to be used in tasks that evaluate the binding of verbal information when assessing the EB.

Additionally, this discrepancy indicates the need to further investigate the binding of verbal material. For example, binding might be performed automatically when linguistic information is available, such as with stories, words in sentences, and constrained sentences, but it draws on executive resources in more arbitrary tasks, such as in the chunking of unrelated sentences and chunking of words in clustered word lists (Baddeley, 2012).

\section{Crossmodal tasks}

The tasks used to assess crossmodal binding in WM involved connections between verbal, visual, and spatial features. In the implementation of the tasks, the stimulus exposure times ranged from 1,000 to 5,000 ms (Table 2), with some studies using a minimum time sufficient for coding. Generally, exposure times in crossmodal tasks were longer than in unimodal tasks (which presented stimuli for durations as short as 250 $\mathrm{ms}$ ), possibly because the stimulus to be encoded (e.g., verbal information and spatial locations) in crossmodal tasks requires slightly more presentation time to be encoded and is thus more attentionally demanding (Holcombe \& Cavanagh, 2001). The RI varied between 950 and 20,000 ms (Table 2), with intervals generally longer than those used in unimodal tasks (Table 1; but see Ally et al., 2008; Jefferies et al., 2004). Crossmodal recall tasks did not adopt RIs (Table 2). When the tasks involved delays longer than a few seconds (e.g., 1-3 s), the likelihood that attention was diverted because of endogenous or exogenous distractions increased, despite the fact that attention can also be diverted within a short time. Moreover, longer delays might demand support from LTM for learning (for a review, see Jeneson \& Squire, 2012).

Crossmodal tasks are hypothesized to require that the features are actively bound in the EB, thus demanding more executive resources, especially when spatial location is a feature to be bound (Allen et al., 2012). This might be attributable to the involvement, in memory for locations, of specialized processes and levels of representation that are distinct from those involved in the processing of surface features of objects (Prabhakaran, Narayanan, Zhao, \& Gabrieli, 2000; Treisman \& Gelade, 1980; Treisman \& Zhang, 2006), as observed in imaging studies (Prabhakaran et al., 2000). The binding of verbal and visuospatial information involves both the phonological loop and visuospatial sketchpad, which feed information to the EB, where it is then bound into single representations (i.e., chunks). The binding of information provided by both slave systems thus appears to correspond to the active binding suggested by Baddeley (2007, p. 172).

Two studies investigated the hypothesis that crossmodal binding requires attentional resources. In the study by Elsley and Parmentier (2009), the participants were not explicitly instructed to maintain the bindings, 
in contrast to other studies, which, according to the authors, may have led to a combination of controlled and automatic binding. However, responses in the concurrent task in this study were obtained after the memory judgments, and the tone maintenance task could have disrupted the response phase (c.f., Allen et al., 2006; Allen, Hitch, \& Baddeley, 2009; Karlsen et al., 2010). Allen et al. (2012) suggested that deciding whether a test probe is composed of the pairing of features that were encountered together originally in crossmodal tasks is more difficult or attentionally demanding than the simple feature judgments in unimodal tasks. Thus, the response, and not the generation or maintenance of bindings, could be impaired by a concurrent task (Baddeley et al., 2011). Some studies indicated that underlying processes may vary across the type of response required in the test phase (e.g., Alvarez \& Thompson, 2009; Hyun, Woodman, Vogel, Hollingworth, \& Luck, 2009).

Similarly, Godoy and Galera (2011) used a task in which the same type of response was emitted, but they used human faces as the stimuli, whose complexity is considerably greater because they have more perceptive details. Faces are thus likely to require more attentional resources to be processed. Additionally, a concurrent BCT task was used that demands more executive resources than those used in other studies (e.g., Allen et al., 2009).

In summary, few studies investigated the role of attentional resources in crossmodal binding compared with unimodal binding. The two studies that investigated this subject exhibited methodological differences, such as the use of highly attentionally demanding secondary tasks and complex stimuli, which makes comparisons of their results with other unimodal tasks difficult. Therefore, we suggest that it is necessary to investigate whether crossmodal binding is affected by attentional load in tasks with less complex stimuli and less attentionally demanding secondary tasks. Additionally, it is unclear whether crossmodal binding per se demands executive resources or if the type of response is affected by a secondary task. The investigation of these problems could contribute to specifying the types of binding involved in specific tasks.

\section{Standardized tests}

Although many of the studies used standardized tests to assess EB function, none of them attempted to provide evidence that the EB is recruited during the execution of such tasks, instead adopting the premise that these tests are appropriate for assessing the EB. Moreover, few of the studies offered a theoretical explanation of why this component would be recruited.

Henry (2010) used the verbal tests Memory for Stories, Paired Recall, and Category Fluency (semantic) to evaluate the EB. All of these tasks were hypothesized to demand the binding of information from different sources, specifically information from phonological short-term memory and activated semantic and linguistic knowledge from LTM, thus requiring contributions from the EB. The authors did concede, however, that all these tasks were indirect measures of the EB.

Petruccelli et al. (2012) used the Recalling Sentences task as a measure of EB function based on suggestions from previous reports (Alloway, Gathercole, Willis, \& Adams, 2004; Baddeley, 2000). Indeed, Alloway et al. (2004) employed a similar task, one that was not part of any standardized test. Both Alloway et al. (2004) and Petruccelli et al. (2012) justified the use of sentence recall in a similar way as Henry (2010), asserting that it demands the binding of phonological information in the phonological loop with semantic and syntactic information from LTM, which should be conducted by the EB. In fact, although Baddeley (2012) recently suggested that the binding of words into chunks is performed automatically in LTM instead of in the $\mathrm{EB}$, the EB is regarded as a structure where bound information can be displayed and is available for further manipulation.

Tests that evaluate the memory of stories are frequently used as measures of the EB (Altgassen et al., 2007; Baddeley \& Wilson, 2002; Berlingeri et al., 2008; Gooding et al., 2005; Henry, 2010). Delayed retrieval relies on long-term episodic memory, but immediate retrieval may depend on the EB (Tudesco et al., 2010). According to Baddeley and Wilson (2002), the immediate retrieval of stories requires not only that the relevant representations be preserved but also that executive processes are available to combine them into novel episodes and maintain those episodes in the EB.

Although Tudesco et al. (2010) also considered immediate logical memory a measure of the EB, they suggested that the operation span test is a more specific test for evaluating the EB. This test requires the subjects to solve mathematical operations while trying to remember words. In this task, the subject is forced to store material in WM and engage executive attention processes (Conway, Kane, Bunting, Hambrick, Wilhelm, \& Engle, 2005). Thus, the secondary task (i.e., solving mathematical operations) prevents the subjects from using memory strategies to increase the number of items (words) recalled. Therefore, the span measured while the subject is performing any task that prevents rehearsal would reflect the capacity of the EB more accurately, which was also discussed by Turner and Engle (1989). Baddeley (2007, p. 192) suggested that the operation span task provides a measure of WM span and stated (p. 150) that this span reflects the storage capacity of the EB. Nevertheless, the specificity of this task in evaluating the EB is unclear because WM span may draw on other components of WM. Indeed, Baddeley (2007, p. 185) suggested that it is also influenced by the central executive and phonological loop.

Tasks that require executive function, namely semantic fluency ("animals," "clothing," and "items from the supermarket") and phonemic fluency (letters F-A-S), were also used by Price et al. (2010), who considered them satisfactory measures of EB function. According to these authors, the verbal fluency task 
demands strategic access to LTM, the temporary storage of information, and the integration of multimodal information. The exact way in which verbal fluency tasks demand the special integration of multimodal information was not clarified by the authors, with the task appearing to have been chosen because it demands access to long-term memory.

The Paired Recall task from the TOMAL is hypothesized to demand contributions from the phonological loop, the central executive, information in LTM about word meanings and word associations, and the integration of that information by the EB (Henry, 2010). With regard to the CVLT and RAVLT, Allen et al. (2010) and Martins and Ortiz (2009) considered that individuals use both the phonological loop and EB during delayed recall to retrieve the material stored in LTM. The first learning trial and distractor trial in the CVLT and BFLT-E were used in the study by Allen et al. (2010). These measures were selected because they were supposed to reflect short-term memory processing and LTM processing, respectively, the integration of which is dependent on the EB. The recall task employed by Allen et al. (2010) was not specified, but their explanation points to the use of delayed recall. Martins and Ortiz (2009) explicitly stated that delayed recall was the task used in their study. The entry of new material into episodic LTM is assumed to depend on the EB. This is supported by Baddeley (2007, pp. 171-172), who suggested that the EB might play an important role in episodic retrieval.

Based on these studies, all of the standardized tests used in these studies appear to require the integration of information in short- and long-term memory. Moreover, verbal tests were more frequently employed than visual tests. Notably, a need exists for evidence that these tests indeed place differential demands on the EB. The tests used in the studies reviewed herein preceded the proposal of the EB by Baddeley (2000), and, therefore, were not created with the purpose of evaluating this component of WM. The tasks involved in these instruments usually recruit a broader scope of functions than those performed by the EB, having been conceived as tests of other cognitive abilities, such as executive function and other components of WM. The adequacy of these tasks for the evaluation of the EB is thus a problem that should be empirically addressed, perhaps by correlating measures of experimental studies with scores on standardized tests to verify the similarity between the cognitive processes recruited to resolve the tasks.

According to Baddeley (2000), because the procedures involved in these tests are rarely processpure, experimental designs that include the use of secondary tasks to study phonological and visuospatial memory and the central executive should allow researchers to ascertain the contributions of each of these systems to the tasks. The experimental tasks developed in the studies reviewed herein make a contribution in this regard. However, the construction of standardized tests that differentially assess the functions of the EB is still necessary.

\section{Conclusion}

A review of the tasks used to assess EB function shows heterogeneity in the ways in which that aim might be accomplished. However, for some of the tasks, it is not always clear which process of the EB is being evaluated. In the case of standardized tests, such subprocesses are rarely specified, whereas the experimental tasks appear to make that point more explicit.

Some precautions should be taken by researchers when designing tasks for the assessment of the EB. First, the delay between the study and test phases should not exceed the limit of short-term retention. Second, in studies that investigate the role of attention in crossmodal binding, researchers should use secondary tasks that employ material in different modalities than the primary task. This methodological strategy might help to avoid the effects of the secondary task being explained in terms of their interference with the same storage components recruited by the primary task. Third, deciding whether the participants should be explicitly instructed to maintain bindings is important. This is attributable to the fact that when this instruction is not explicitly given, recovery processes might involve a combination of automatic and controlled processes (Elsley \& Parmentier, 2009). Thus, instructing participants should minimize this combination.

The evaluation of the EB and its subprocesses could benefit from the construction of tasks and experimental paradigms that are able to assess distinct binding processes. This experimental approach is still necessary because of the lack of agreement concerning the processes in the EB in terms of its specificity in dealing with different information and the relationships between different processes.

\section{References}

References marked with (•) indicate studies included in this systematic review.

- Allen, D. N., Randall, C., Bello, D., Armstrong, C., Frantom, L., Cross, C., \& Kinney, J. (2010). Are working memory deficits in bipolar disorder markers for psychosis? Neuropsychology, 24(2), 244-254.

- Allen, R. J., Baddeley, A. D., \& Hitch, G. J. (2006). Is the binding of visual features in working memory resource-demanding? Journal of Experimental Psychology: General, 135(2), 298-313.

Allen, R. J., Hitch, G. J., \& Baddeley, A. D. (2009). Cross-modal binding and working memory. Visual Cognition, 17(1-2), 83-102.

- Allen, R. J., Hitch, G. J., Mate, J., \& Baddeley, A. D. (2012). Feature binding and attention in working memory: a resolution of previous contradictory findings. Quarterly Journal of Experimental Psychology, 65(12), 2369-2383.

- Alloway, T. P., Gathercole, S. E., Willis, C., \& Adams, A. M. (2004). A structural analysis of working memory and related cognitive skills in young children. Journal of Experimental Child Psychology, 87(2), 85-106.

- Ally, B. A., Simons, J. S., McKeever, J. D., Peers, P.V., \& Budson, A. E. (2008). Parietal contributions to recollection: electrophysiological evidence from aging and patients with parietal lesions. Neuropsychologia, 46(7), 1800-1812.

- Altgassen, M., Phillips, L., Kopp, U., \& Kliegel, M. (2007). Role of working memory components in planning performance of 
individuals with Parkinson's disease. Neuropsychologia, 45(10), 2393-2397.

Alvarez, G. A., \& Thompson, T. W. (2009). Overwriting and rebinding: why feature-switch detection tasks underestimate the binding capacity of visual working memory. Visual Cognition, 17(1-2), 141-159.

Baddeley, A. D. (1966). Short-term memory for word sequences as a function of acoustic, semantic and formal similarity. Quarterly Journal of Experimental Psychology, 18(4), 362-365.

Baddeley, A. D. (1996). Exploring the central executive. The Quarterly Journal of Experimental Psychology, 49A, 5-28.

Baddeley, A. D. (2000). The episodic buffer: a new component of working memory? Trends in Cognitive Sciences, 4(11), 417-423.

Baddeley, A. D. (2007). Working memory, thought, and action. New York: Oxford University Press.

Baddeley, A. D. (2012). Working memory, theories models and controversies. Annual Review of Psychology, 63, 1-29.

Baddeley, A. D., Allen, R. J., \& Hitch, G. (2010). Investigating the episodic buffer. Psychologica Belgica, 50(3-4), 223-243.

- Baddeley, A. D., Allen, R. J., \& Hitch, G. J. (2011). Binding in visual working memory: the role of the episodic buffer. Neuropsychologia, 49, 1393-1400.

Baddeley, A. D., \& Hitch, G. (1974). Working memory. In G.H. Bower (Ed.), The psychology of learning and motivation: advances in research and theory (vol. 8, pp. 47-89). New York: Academic Press.

- Baddeley, A. D., Hitch, G. J., \& Allen, R. J. (2009). Working memory and binding in sentence recall. Journal of Memory and Language, 61(3), 438-456.

Baddeley, A. D., \& Logie, R. H. (1999). Working memory: the multiple component model. In A. Miyake, \& P. Shah (Eds.), Models of working memory: mechanisms of active maintenance and executive control (pp. 28-61). New York: Cambridge University Press.

Baddeley, A. D., \& Wilson, B. A. (2002). Prose recall and amnesia: implications for the structure of working memory. Neuropsychologia, 40(10), 1737-1743.

- Belacchi, C., Benelli, B., \& Pantaleone, S. (2011). The influence of categorical organization on verbal working memory. British Journal of Developmental Psychology, 29(Pt 4), 942-960.

- Berlingeri, M., Bottini, G., Basilico, S., Silani, G., Zanardi, G., Sberna, M., Colombo, N., Sterzi, R., Scialfa, G., \& Paulesu, E. (2008). Anatomy of the episodic buffer: a voxel-based morphometry study in patients with dementia. Behavioural Neurology, 19(1-2), 29-34.

Brandimonte, M. A., Hitch, G. J., \& Bishop, D. V. (1992). Verbal recoding of visual stimuli impairs mental image transformations. Memory and Cognition, 20(4), 449-455.

- Campo, P., Maestú, F., Capilla, A., Morales, M., Fernández, S., del Río, D., \& Ortiz, T. (2008). Temporal dynamics of parietal activity during word-location binding. Neuropsychology, 22(1), 85-99.

Conway, A. R. A., Kane, M. J., Bunting, M. F., Hambrick, D. Z., Wilhelm, O., \& Engle, R. W. (2005). Working memory span tasks: a methodological review and user's guide. Psychonomic Bulletin \& Review, 12, 769-786.

Daneman, M., \& Carpenter P. A. (1980). Individual differences in working memory and reading. Journal of Verbal Learning and Verbal Behavior, 19, 450-466.

- Elsley, J. V., \& Parmentier, F. B. R. (2009). Is verbal-spatial binding in working memory impaired by a concurrent memory load? Quarterly Journal of Experimental Psychology, 62(9), 1696-1705.

- Germano, C., Kinsella, G. J., Storey, E., Ong, B., \& Ames, D. (2008). The episodic buffer and learning in early Alzheimer's disease. Journal of Clinical and Experimental Neuropsychology, 30(6), 627-638.

- Glosser, G., Cole, L., Khatri, U., DellaPietra, L., \& Kaplan, E. (2002). Assessing nonverbal memory with the Biber Figure Learning TestExtended in temporal lobe epilepsy patients. Archives of Clinical Neuropsychology, 17(1), 25-35.

- Godoy, J. P. M. C., \& Galera, C. (2011). Binding faces and names in working memory requires additional attentional resources. Psychology \& Neuroscience, 4(3), 341-346.

- Gooding, P. A., Isaac, C. L., \& Mayes, A. R. (2005). Prose recall and amnesia: more implications for the episodic buffer. Neuropsychologia, 43(4), 583-587.
- Harkin, B., \& Kessler, K. (2009). How checking breeds doubt: reduced performance in a simple working memory task. Behaviour Research and Therapy, 47(6), 504-512.

- Henry, L. A. (2010). The episodic buffer in children with intellectual disabilities: an exploratory study. Research in Developmental Disabilities, 31(6), 1609-1614.

- Hoffman, P., Jefferies, E., Ehsan, S., Jones, R. W., \& Lambon Ralph, M. A. (2012). How does linguistic knowledge contribute to shortterm memory? Contrasting effects of impaired semantic knowledge and executive control. Aphasiology, 26(3-4), 383-403.

Holcombe, A. O., \& Cavanagh, P. (2001). Early binding of feature pairs for visual perception. Nature Neuroscience, 4(2), 127-128.

Hyun, J., Woodman, G. F., Vogel, E. K., Hollingworth, A., \& Luck, S. J. (2009). The comparison of visual working memory representations with perceptual inputs. Journal of Experimental Psychology: Human Perception and Performance, 35(4), 1140-1160.

- Jefferies, E., Lambon Ralph, M. A., \& Baddeley, A. D. (2004). Automatic and controlled processing in sentence recall: the role of long-term and working memory. Journal of Memory and Language, 51(4), 623-643.

Jeneson, A., \& Squire, L. R. (2012). Working memory, long-term memory, and medial temporal lobe function. Learning \& Memory, 19, 15-25.

- Kapikian, A., \& Briscoe, J. (2012). Semantic binding, not attentional control, generates coherent global structure in children's narrative memory. European Journal of Cognitive Psychology, 24, 751-764.

- Karlsen, P. J., Allen, R. J., Baddeley, A. D., \& Hitch, G. J. (2010). Binding across space and time in visual working memory. Memory \& Cognition, 38(3), 292-303.

- Kessels, R. P. C., Meulenbroek, O., Fernández, G., \& Olde Rikkert, M. G. M. (2010). Spatial working memory in aging and mild cognitive impairment: effects of task load and contextual cueing. Aging, Neuropsychology, and Cognition, 17(5), 556-574.

- Luck, D., Danion, J. M., Marrer, C., Pham, B. T., Gounot, D., \& Foucher, J. (2010a). The right parahippocampal gyrus contributes to the formation and maintenance of bound information in working memory. Brain and Cognition, 72(2), 255-263.

- Luck, D., Danion, J.M., Marrer, C., Pham, B. T., Gounot, D., \& Foucher, J. (2010b). Abnormal medial temporal activity for bound information during working memory maintenance in patients with schizophrenia. Hippocampus, 20(8), 936-948.

- Martins, F. C., \& Ortiz, K. Z. (2009). The relationship between working memory and apraxia of speech. Arquivos de NeuroPsiquiatria, 67(3B), 843-848.

- Mate, J., Allen, R. J., \& Baqués, J. (2012). What you say matters: exploring visual-verbal interactions in visual working memory. Quarterly Journal of Experimental Psychology, 65(3), 395400.

Miller, G. A. (1956). The magical number seven, plus or minus two: some limits on our capacity for processing information. Psychological Review, 63(2), 81-97.

- Newman, S. D., Malaia, E., Seo, R., \& Cheng, H. (2012). The effect of individual differences in working memory capacity on sentence comprehension: an fMRI study. Brain Topography, in press.

- Parra, M. A., Sala, S. D., Logie, R. H., \& Abrahams, S. (2009). Selective impairment in visual short-term memory binding. Cognitive Neuropsychology, 26(7), 583-605.

Petruccelli, N., Bavin, E.L., \& Bretherton, L. (2012). Children with specific language impairment and resolved late talkers: working memory profiles at 5 years. Journal of Speech, Language, and Hearing Research, 55(6), 1690-1703.

Phillips, W. A., \& Baddeley, A. D. (1971). Reaction time and shortterm visual memory. Psychononic Science, 22(2), 73-74.

- Piolino, P., Coste, C., Martinelli, P., Macé, A.L., Quinette, P., Guillery-Girard, B., \& Belleville, S. (2010). Reduced specificity of autobiographical memory and aging: do the executive and feature binding functions of working memory have a role? Neuropsychologia, 48(2), 429-440.

- Poch, C., Campo, P., Parmentier, F. B. R., Ruiz-Vargas, J. M., Elsley, J. V., Castellanos, N. P., Maestú, F., \& del Pozo, F. (2010). Explicit processing of verbal and spatial features during letter-location binding modulates oscillatory activity of a fronto-parietal network. Neuropsychologia, 48(13), 3846-3854. 
Prabhakaran, V., Narayanan, K., Zhao, Z., \& Gabrieli, J. D. E. (2000). Integration of diverse information in working memory within the frontal lobe. Nature Neuroscience, 3, 85-90.

- Price, S. E., Kinsella, G. J., Ong, B., Mullaly, E., Phillips, M., Pangnadasa-Fox, L., Perre, D., \& Storey, E. (2010). Learning and memory in amnestic mild cognitive impairment: contribution of working memory. Journal of the International Neuropsychological Society, 16, 342-351.

- Quinette, P., Guillery-Girard, B., Noël, A., de la Sayette, V., Viader, F., Desgranges, B., \& Eustache, F. (2006). The relationship between working memory and episodic memory disorders in transient global amnesia. Neuropsychologia, 44(12), 2508-2519.

Repovs, G., \& Baddeley, A. D. (2006) Multi-component model of working memory: explorations in experimental cognitive psychology. Neuroscience, 139, 5-21.

- Rudner, M., Fransson, P., Ingvar, M., Nyberg, L., \& Rönnberg, J. (2007). Neural representation of binding lexical signs and words in the episodic buffer of working memory. Neuropsychologia, 45(10), 2258-2276.
Strauss, E., Sherman, E. M. S., \& Spreen, O. (2006). A compendium of neuropsychological tests: administration, norms, and commentary, 3rd edition. New York: Oxford University Press.

Treisman, A., \& Gelade, G. (1980). A feature-integration theory of attention. Cognitive Psychology, 12, 97-136.

Treisman, A., \& Zhang, W. (2006). Location and binding in visual working memory. Memory \& Cognition, 34(8), 1704-1719.

- Tudesco, I. S., Vaz, L. J., Mantoan, M. A. S., Belzunces, E., Noffs, M. H., Caboclo, L. O. S. F., Yacubian, E. M. T., Sakamoto, A. C., \& Bueno, O. F. A. (2010). Assessment of working memory in patients with mesial temporal lobe epilepsy associated with unilateral hippocampal sclerosis. Epilepsy \& Behavior, 18(3), 223-228.

Turner, M. L., \& Engle, R. W. (1989). Is working memory capacity task dependent? Journal of Memory and Language, 28, 127-154.

- Vaz, L. J., Pradella-Hallinan, M., Bueno, O. F. A., \& Pompéia, S. (2011). Acute glucocorticoid effects on the multicomponent model of working memory. Human Psychopharmacology, 26(7), 477-487.

-Zhang, D., Zhang, X., Sun, X., Li, Z., Wang, Z., He, S., \& Hu, X. (2004). Cross-modal temporal order memory for auditory digits and visual locations: an fMRI study. Human Brain Mapping, 22(4), 280-289. 